\title{
CDISC SDTM Study Type Terminology
}

National Cancer Institute

\section{Source}

National Cancer Institute. CDISC SDTM Study Type Terminology. NCI Thesaurus. Code C99077.

Terminology associated with the study type codelist of the Clinical Data Interchange Standards Consortium (CDISC) Study Data Tabulation Model (SDT M). 